\section{Prädiktive Biomarker beim Rektumkarzinom}

\author{
Bei Patienten mit lokal fortgeschrittenem Rektumkarzinom stellt sich \\ die Frage, wie es nach totaler mesorektaler Exzision weitergeht. Die \\ Erfolgschancen der adjuvanten Chemoradiotherapie sind nicht so \\ leicht einzuschätzen. Neue Biomarker könnten hier hilfreich sein.
}

U m das Risiko einer Fernmetastasierung nach der Tumorresektion und vor der anschließenden adjuvanten Chemoradiotherapie zu beurteilen, gelten T-Stadium, Lymphknotenbefall und Status des zirkumferenziellen Resektionsrandes als besonders wichtig. Allerdings unterscheiden sich die Behandlungsergebnisse oft erheblich, auch wenn die Patienten hinsichtlich dieser prognostischen Faktoren vergleichbar sind. Grund genug, nach zusätzlichen molekularen Markern für das Metastasierungsrisiko zu suchen.

Das geschah bei 68 Patienten mit lokal fortgeschrittenem Adenokarzinom des Rektums, die sich einer totalen mesorektalen Resektion und dann einer adjuvanten Chemoradiotherapie (6-12 Zyklen 5-Fluorouracil plus Folinsäure, 54 Gy in 30 Fraktionen) unterzogen hatten. Das resezierte Tumorgewebe analysierte man immunhistochemisch auf fünf verschiedene molekulare Marker: Cyclooxygenase (COX)-2, epidermaler Wachstumsfaktor-Rezeptor (EGFR), vaskulärer endothelialer Wachstumsfaktor (VEGF), Thymidinsynthase (TS) und Raf-Kinase-Inhibitor-Protein (RKIP).

Während einer medianen Beobachtungszeit von 65 Monaten traten acht lokoregionäre Rezidive $(11,8 \%)$ und 13 Fernmetastasen (19,1\%) auf. In der multivariaten Analyse erwiesen sich positive Befunde für COX-2 und VEGF sowie negative Ergebnisse für RKIP als unabhängige prognostische Faktoren für das Überleben frei von Fernmetastasen, das krankheitsfreie und das Gesamtüberleben. Die Kombination COX-2+/VEGF+ korrelierte am stärksten mit einem verminderten krankheitsfreien Überleben, verglichen mit dem Rest der Patienten, RKIP+/COX-2- und RKIP+/VEGF- am stärksten mit einem verbesserten krankheitsfreien Überleben. Für EGFR und TS fand sich dagegen kein Bezug zu Rezidiv oder Überleben.
Fazit: Molekulare Marker wie COX-2+, VEGF+ und RKIP- signalisieren bei Patienten mit lokal fortgeschrittenem Rektumkarzinom ein erhöhtes Risiko für Fernmetastasen nach adjuvanter Chemoradiotherapie. Das könnte zu einer besser auf den Einzelfall zugeschnittenen Behandlung beitragen, beispielsweise einer intensivierten Erhaltungschemotherapie bei Hochrisikopatienten.

Ulrike Wepner

Kim JW et al. Molecular markers predict distant metastases after adjuvant chemoradiation for rectal cancer. Int J Radiat Oncol Biol Phys. 2012;84(5):e577-84.

Kommentar von Prof. Hiller: Bei dieser retrospektiven Untersuchung von 68 Patienten hatten $91,2 \%$ ein pT3-Stadium und $57,4 \%$ ein pN1- oder pN2-Stadium. Ab Stadium T3, N0 bzw. T1 oder T2 mit Lymphknotenbefall wäre z. B. nach den Richtlinien des Tumorzentrums München eine kombinierte neoadjuvante Radiochemotherapie mit nachfolgender Rektumresektion vorgesehen. In der hier beschriebenen Patientenkohorte erfolgte zunächst die totale mesorektale Resektion und nachfolgend eine adjuvante Chemoradiotherapie. Insofern unterschied sich das Vorgehen der koreanischen Arbeitsgruppe von unserem derzeitigen Vorgehen. Nun führt sowohl die neoadjuvante wie auch die adjuvante Chemoradiotherapie für die betroffenen Patienten neben der Operation zu einer zusätzlichen hohen Belastung mit oft lang anhaltenden Folgeschäden (Urogenitalsystem, Darm). Wir wissen, dass nach fünf Jahren nur ein Teil der Patienten ein Lokalrezidiv oder eine Fernmetastasierung erleiden wird, der größere Teil wird "geheilt" sein, obwohl die Patienten initial ein vergleichbares T- und $\mathrm{N}$ - Stadium sowie einen vergleichbaren Status des Resektionsrandes aufwiesen. Die Frage, die sich nun den Autoren stellte, war, ob es neben dem Tumor- und Lymphknotenstatus sowie dem Sicherheitsabstand des Resektionsrandes auch molekulare Marker gibt, welche eine prognostische
Aussage zum Risiko eines Progresses der Erkrankung erlauben. Die hier immunhistochemisch untersuchten molekularen Marker COX-2, EGFR, VEGF und RKIP spielen bei einer Vielzahl von Tumoren eine wichtige Rolle bei Tumorwachstum und -progression. Die Thymidinsynthase ist ein wichtiges Target für die Chemotherapie und das in diesem Zusammenhang eingesetzte 5-Fluorouracil. Im Beobachtungszeitraum von fünf Jahren kam es bei $19 \%$ der Patienten zur Fernmetastasierung, bei 11,8\% zum lokoregionären Rezidiv. In der multivariaten Analyse erwiesen sich COX-2-positive und VEGF-positive Befunde und RKIP-negative Befunde als unabhängige prognostische Faktoren für das verminderte krankheitsfreie und gesamte Überleben, die untersuchten EGFR und TS spielten in diesem Zusammenhang keine Rolle. Das Bemühen der Autoren in dieser Analyse die Rolle der molekularen Marker als Prognosefaktoren zu evaluieren, ist sicher begrüßenswert. Aufgrund der niedrigen Fallzahl und der Natur der retrospektiven Analyse kann diese Kommunikation derzeit jedoch nur als Anreiz verstanden werden, der Fragestellung in einer prospektiven Studie mit höherer Fallzahl weiter nachzugehen. Aber vielleicht liegt die Zukunft in den sogenannten diagnostischen Gentests. Beim Hormonrezeptorpositiven Mammakarzinom (das sind $75 \%$ aller Mammakarzinome) wird schon vielerorts der Oncotype DX ${ }^{\circledR}$-Test (bestehend aus 21 Genen) zur Unterstützung der Behandlungsentscheidung eingesetzt, d. h. er hilft bei der Entscheidung, ob zusätzlich zur Hormontherapie eine Chemotherapie erforderlich ist. Die Hersteller (Genomic Health, INC) haben auch einen Test für Darmkrebspatienten entwickelt. Mithilfe des Oncotype DX ${ }^{\circ}$ Colon Cancer Assay wird derzeit die Aktivität von krebsspezifischen Genen in Gewebsproben eines Darmtumors untersucht. Vielleicht sieht die Zukunft so aus, dass auch bei Patienten mit kolorektalen Tumoren ein Gentest zur Therapieentscheidung über eine notwendige oder auch entbehrliche neoadjuvante oder adjuvante Radiochemotherapie beitragen kann.

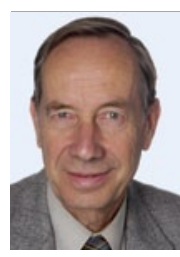

Prof. Dr. med. Erhard Hiller Häamto-onkologische Praxis, München erhard.hiller@gmx.de 